\title{
Pratiques
}

Linguistique, littérature, didactique

$169-170 \mid 2016$

Enseignement/apprentissage de la langue, des textes et des discours. 40\&nbspans de Pratiques

\section{Didactique de l'étymologie au collège : un exemple de relations entre recherche fondamentale et recherche-action}

Teaching Etymology to Young Learners: Connecting Linguistics and Action

Research

\section{Francis Carton et Dominique Macaire}

\section{(2) OpenEdition}

Journals

Édition électronique

URL : http://journals.openedition.org/pratiques/3020

DOI : $10.4000 /$ pratiques.3020

ISSN : 2425-2042

Éditeur

Centre de recherche sur les médiations (CREM)

Référence électronique

Francis Carton et Dominique Macaire, « Didactique de l'étymologie au collège : un exemple de relations entre recherche fondamentale et recherche-action », Pratiques [En ligne], 169-170 | 2016, mis en ligne le 30 juin 2016, consulté le 10 décembre 2020. URL : http://journals.openedition.org/ pratiques/3020 ; DOI : https://doi.org/10.4000/pratiques.3020

Ce document a été généré automatiquement le 10 décembre 2020.

(c) Tous droits réservés 


\title{
Didactique de l'étymologie au collège : un exemple de relations entre recherche fondamentale et recherche-action
}

\author{
Teaching Etymology to Young Learners: Connecting Linguistics and Action \\ Research
}

Francis Carton et Dominique Macaire

1 La recherche en didactique des langues (DDL) se voit considérée par certains comme un parent pauvre de la recherche fondamentale, sous prétexte que la recherche de terrain, et la recherche-action notamment, n'apporteraient pas de connaissances stricto sensu et ne contribuerait ainsi pas à la recherche fondamentale dont c'est l'objet. Longtemps on a opposé la recherche fondamentale à la recherche dite "appliquée ", surtout dans les domaines des sciences dures. Notre point de vue est différent: pour nous, la DDL constitue l'un des domaines des sciences du langage. Son angle propre, par rapport aux autres disciplines, est d'apporter des connaissances sur l'apprentissage des langues. Une pratique courante de la DDL est de procéder par recherche-action, un processus interventionniste : pour apporter des changements dans le monde éducatif, elle cherche à pénétrer les pratiques des différents acteurs au plus près de leur complexité. En ce sens, elle est contributive des recherches fondamentales.

2 Nous montrerons qu'une approche renouvelée de l'étymologie romane, mise au point par des spécialistes en linguistique historique, est transposable à l'école, et qu'elle est utile pour les élèves en ce qu'elle peut contribuer à rendre plus justes leurs représentations sur les langues. Les expérimentations ont eu lieu auprès d'élèves de collège en France en 2013-2014. Elles ont donné lieu à une description dans D. Macaire et al. (2014), et le protocole est disponible en ligne (http://weblettres.net/pedagogie/index2.php? rub=14\&ssrub=57 ; adresse contact : enseignement-etymologie@atilf.fr). 
3 Nous ne cherchons pas dans cet article à mesurer les acquisitions des élèves, à examiner des modalités d'acquisition, ou à évaluer des conduites d'enseignement. Notre intérêt porte ici sur la manière dont les enseignants et les collégiens s'approprient une démarche nouvelle, établie par des chercheurs en linguistique, et sur ce que celle-ci peut apporter aux élèves. Les enjeux de l'expérience visent moins l'acquisition de connaissances sur des langues que l'accès à un ensemble de représentations: le français appartient à la famille des langues romanes, et il est possible de reconstruire l'histoire d'un mot par la comparaison entre les «mots cousins » (cognats) de ces différentes langues. Après avoir décrit en quoi consiste cette approche de l'étymologie romane et les enjeux didactiques que nous y voyons, nous décrirons les expérimentations qui ont été menées et, à partir de l'analyse des données recueillies, nous répondrons aux questions posées.

\section{Un objet de recherche en linguistique historique : le DÉRom}

4 Depuis quelques années, un nouveau paradigme scientifique en étymologie romane a vu le jour. Développé par le laboratoire ATILF, le Dictionnaire Étymologique Roman (DÉRrom: (Buchi, 2010; Buchi \& Schweickard, 2011a, 2011b) repose sur l'idée que la comparaison des mots entre langues romanes permet de reconstruire les étymons protoromans.

5 Le consensus reposait jusqu'alors sur un postulat de base tacitement admis, qu'en raison de l'abondance des témoignages écrits du latin, les étymons du lexique héréditaire roman n'avaient pas besoin d'être reconstruits à travers les opérations de la grammaire comparée mais pouvaient être prélevés dans des dictionnaires latins. C'est ce que l'on appelle la méthode « de la pioche ». Or les mots du français et des autres langues romanes ne viennent pas du langage de Cicéron ou de Tacite, mais de variétés dites "basses", « populaires » ou encore « vulgaires » du latin.

6 Le DÉrom cherche à reconstruire cette base, le protoroman, en utilisant les méthodes de la grammaire comparative-reconstructionniste. Par exemple, la comparaison des mots français nuit, roumain nopŢii, italien notte, catalan nit, espagnol noche, portugais noite permet, grâce aux lois de la phonétique et de la grammaire historique, de reconstruire le protoroman */'nokt-e / (latin nox, gen. noctis). Cette démarche permet à l'étymologie romane de réintégrer les principes de la linguistique historique, et le paradigme accepté pour les études étymologiques des autres familles de langues.

7 En quelques années, cette approche a bénéficié d'un vaste mouvement d'adhésion au sein de la linguistique romane. Cependant, celle-ci n'est pas entière : certaines critiques ont été virulentes, au motif que le paradigme serait «non vulgarisable » (Vàrvaro, 2011a, 2011b), notamment auprès de publics scolaires. Un tel reproche est probablement dû pour partie aux pratiques didactiques couramment à l'œuvre dans le traitement de l'étymologie dans l'institution scolaire. Même si cette critique ne met pas en cause la scientificité du nouveau paradigme, l'ATILF a entrepris de tester cette assertion et de faire valider son option dans le cadre d'une recherche-action: l'hypothèse est que les élèves de collège sont cognitivement en mesure d'approcher l'histoire de la langue française dans sa dimension comparative avec les autres langues romanes. 


\section{Enjeux didactiques}

8 Les équipes Linguistique historique française et romane et Didactique des langues et sociolinguistique de l'ATILF ont mis en place le projet de recherche Etymodidac, qui compte une douzaine de personnes, chercheurs des deux équipes et praticiens en recherche. Les options théoriques du DÉRom ont été confrontées aux orientations didactiques de l'approche plurilingue portées par certains chercheurs de l'équipe Didactique des langues et sociolinguistique, puis « mises en pratiques de classes » au sein d'un protocole proposant la mise en œuvre d'un scénario pédagogique.

9 À l'époque où a été mise en place cette expérimentation, la ministre française de l'Éducation nationale proposait de généraliser l'étude des langues anciennes au collège au sein d'ateliers appelés Enseignements pratiques interdisciplinaires (EPI) ${ }^{1}$, et non plus dans des cours de langue ancienne, latin ou grec. L'approche préconisée des EPI est avant tout culturelle et historique, ce qui confère une place à la réflexion sur la langue et son évolution, bref un espace pour l'enseignement/apprentissage de l'étymologie à une époque où le latin n'est plus une référence pour les élèves.

10 La démarche DÉrom donne lieu d'abord à une vision plus juste de l'histoire du français (les langues romanes ne procèdent pas du latin classique), et elle contribue à un déplacement des représentations sur l'étymologie dans le discours institué.

11 La démarche de comparaison des langues est susceptible d'initier les élèves à la découverte de cette famille de langues et à son histoire. Elle peut les amener à s'intéresser à l'étymologie dans des contextes «textuels » ou en isolant des mots, pour mener des activités de réflexion sur la langue de type language awareness (Hawkins, 1984 ; Moore, 1995). Depuis une quinzaine d'années, des travaux didactiques s'inscrivant dans une acception théorique plurilingue et comparatiste (Candelier, 2003; Coste, Castelloti \& Duverger, 2008 ; Escudé \& Janin, 2008 ; Macaire, 2008 ; Meissner et al., 2004) ont conduit à des expérimentations sur les emprunts, sur l'intercompréhension entre langues romanes ${ }^{2}$ , sur l'éveil/l'éducation aux langues et aux cultures à l'école primaire 3 , pour n'en nommer que certains. D'un point de vue social, les attentes sur la connaissance des langues, les demandes de réflexivité et de mise en relation des diverses langues apprises sont nombreuses, au sein d'une société dans laquelle les mobilités et les échanges sont de plus en plus nombreux, y compris au sein des établissements scolaires : le plurilinguisme des élèves se développe, notamment en raison des flux migratoires que connaissent les sociétés contemporaines.

12 Par ailleurs, la démarche de reconstruction en elle-même parait intéressante. «Savoir d'où vient une langue contribue à la placer, à lui donner une réalité sociale et scolaire » (Macaire \& al., 2014). Les élèves sont amenés à une analyse exploratoire qui permet de remonter à l'étymon commun à partir d'une série de cognats. Le latin n'est pas une fin en soi, voire un point de départ, il est associé aux langues romanes et placé dans une relation de diachronie avec elles. Cette démarche engage une réflexion sur les registres de langue et la question de la norme langagière (même si bien sûr d'autres approches sont possibles dans ce but). Formuler des hypothèses sur le déroulement temporel, avoir des référents dans le temps relève d'une compétence sociale transversale. Cette démarche peut susciter leur curiosité et leur goût pour la langue. 
13 L'engagement dans une entreprise commune dans laquelle les collégiens sont reconstructeurs des connaissances dans un contrat explicite en début de séquence, mobilise des savoirs sur les langues que peuvent avoir les uns et/ou les autres. Il mobilise également des savoir-faire nécessaires aux interactions entre élèves et des savoir-vivre ensemble.

14 Ce qui caractérise la recherche Etymodidac relève d'un enjeu placé sur la reconstruction et la comparaison, un double processus d'apprentissage dynamique qui place l'élève au centre du processus lui-même. Un tel enjeu engage vers des conclusions qui seront toujours des hypothèses ou qui produiront de nouvelles questions. Mais n'est-ce pas le sort de toute recherche scientifique et dans la nature même du savoir? Ceci peut se révéler déstabilisant lorsqu'il s'agit de transposer des savoirs savants en savoirs scolaires, notamment pour les jeunes apprenants. Soulignons par ailleurs d'une part l'illusion qu'il faut «savoir » pour être "bon », c'est à dire que le savoir serait figé, et d'autre part la croyance que l'enseignant est là pour transmettre le savoir, alors que les espaces-temps de l'apprendre ne correspondent pas idéalement aux séances formelles de cours ni même aux interactions privilégiées entre l'enseignant et la classe.

\section{Expérimentation en collège}

Durant deux années, l'approche du DÉRom a été testée auprès de classes de $6^{\mathrm{e}}$ et de $4^{\mathrm{e}}$ dans plusieurs collèges lorrains ${ }^{4}$, pour une cohorte de 170 élèves. Ces classes sont caractérisées par une grande hétérogénéité, tant au plan des âges différents, que des localisations (ville/campagne) ou des recrutements socioculturels (favorisés/socialement défavorisés), élite scolaire/élèves dans la moyenne/élève dits « en difficulté ». Dans l'une des classes expérimentales, 9 élèves sur 10 ne parlent pas français à la maison.

\subsection{Transposition didactique}

Il ne s'agit pas de vulgariser des savoirs savants, mais de les transformer pour qu'ils soient «enseignables» (du côté de l'enseignant) et susceptibles d'être appris par les élèves, en partant de ce qu'ils savent et de ce qui peut présenter un intérêt scolaire et social pour eux : c'est le processus de transposition externe, parce qu'il a lieu à l'extérieur de la classe (Chevallard, 1985).

17 Les opérations de sélection, de transformation, d'interprétation et de réélaboration didactique des savoirs scientifiques, au cœur de notre travail d'intervention, ont fait l'objet d'un travail collectif de l'ensemble de l'équipe de recherche (linguistes, didacticiens, et enseignantes de collège) :

- sélection du document support ;

- choix des mots à étudier pour la découverte des différentes langues romanes (petit et prince pour le titre, reconnaissance de $\underline{\text { ans }} \underline{\text { mois, }} \underline{\text { dormir, }} \underline{\text { six, }}$ nuit, grandes dans les textes en 6 langues romanes et comparaison de ces mots « cousins »);

- reconstruction de l'étymon de ans, dorment, grandes, nom, verbe et adjectif choisis au pluriel parce que la marque en est plus apparente ;

- processus de reconstruction des étymons, autour de questions adaptées aux possibilités d'élèves de collège (voir l'exemple fourni en annexe) ; 
- détermination des termes outils à utiliser en classe (emploi du terme "protoroman ", mais de « cousins » au lieu de " cognats », ou de « mot ancêtre » au lieu de « étymon »);

- élaboration du scénario, qui prévoit non seulement les «savoirs à enseigner ", mais aussi la planification précise des deux séances, et les ressources susceptibles de mobiliser la motivation (affiche, couvertures du roman, fiches de travail). Le Petit Prince, présenté en 6 langues romanes ${ }^{5}$. Cinq activités sont proposées aux classes :

- mise en situation à partir d'une affiche du Petit Prince ;

- activité collective à partir des couvertures et du titre du Petit Prince, dans les six langues : formulation d'hypothèses et reconnaissance des six langues ;

- repérage dans les 6 textes des mots suivants : ans, quand, dormir, mois, grandes, nuit. Travail de groupes, chaque groupe s'occupant de 2 mots. Répartition des découvertes au tableau, reconnaissance des six langues;

- reconstruction collective des termes protoromans pour ans, dormir et grandes, sous la conduite de l'enseignant ;

- synthèse-structuration permettant d'asseoir les apprentissages et les nouveaux savoirs issus des deux séances.

\subsection{Collecte des données d'expérimentation}

21 Des données croisées ont été recueillies dans les diverses classes : a/ un questionnaire anonyme rempli par les élèves; $b /$ des entretiens semi-dirigés auprès de chaque enseignant à la fin de la deuxième séance; c/ des observations des classes menées conjointement par deux chercheurs issus de chaque équipe de recherche (le DÉRom et l'équipe Didactique des langues); d/ la captation vidéo d'une séance, afin de pouvoir analyser avec davantage de recul les pratiques scolaires à l'œuvre; e/ une séance (enregistrée et transcrite) de "débriefing " de deux heures avec les chercheurs et les enseignants.

\section{Résultats de la recherche}

\subsection{Que faut-il savoir enseigner?}

Les enseignantes expérimentatrices, désignées par leur hiérarchie (Inspectrice pédagogique régionale), n'ont pas participé aux travaux de préparation des deux séances, sauf pour une d'entre elles. Lors d'une formation préalable de trois heures, elles ont pris 
connaissance de l'ensemble du scénario, qui leur a été expliqué en détail, et elles ont reçu des fiches nécessaires à la mise en pratiques. Sous l'injonction de l'institution, elles s'engagent ainsi dans «l'aventure ». Au départ, elles se sont montrées inquiètes, voire paniquées: «j'avais peur », «je n'avais pas tout compris», «c'était quelque chose de nouveau », « cet énorme pavé, je ne le comprenais pas », « c'était hermétique »", etc.

Elles ont préparé leurs séances, en y consacrant plusieurs soirées, et parfois en sollicitant leurs conjoints comme cobayes. Selon leurs dires, ce travail de préparation était destiné moins à transformer les savoirs qui leur ont été donnés à enseigner pour les adapter à leurs élèves, qu'à les comprendre, à les mémoriser, et à anticiper les pratiques pédagogiques à mettre en place.

Lors de la séance de débriefing, quelques jours après la deuxième séance, elles évoquent d'abord leur satisfaction: pour elles, l'expérience est réussie, les objectifs sont globalement atteints. Même s'il existe, suivant les élèves et les niveaux de classes, un écart entre les "savoirs enseignés » et les "savoirs appris ", elles ont le sentiment que tous les élèves ont retiré des éléments utiles de l'expérimentation.

Mais elles parlent aussi des peurs et des angoisses ressenties par elles pendant les deux séances, qu'elles expliquent par une difficulté par rapport au savoir : «stress », « manque d'assurance », « panique », « je ne leur envoie pas l'énergie qu'ils ont l'habitude d'avoir de ma part »; «j'étais différente ». Elles se lancent en effet pour la première fois dans un scénario portant sur des connaissances dont elles disent qu'elles sont mal maîtrisées par elles : « on était accrochées aux feuilles/fiches». Si elles disent avoir compris le sens des apprentissages proposés, certaines ressentent des difficultés à assimiler le scénario dans sa planification et ses objectifs. Ces propos montrent que leur formation à l'étymologie fait défaut, et que leurs connaissances de la linguistique historique leur paraissent trop réduites. Si elles ont toutes eu des cours sur ce domaine à l'université, elles se demandent comment reformuler des savoirs trop peu maîtrisés, et s'accordent à dire qu'un certain niveau de connaissance disciplinaire est utile pour enseigner.

On peut comprendre de ce fait qu'elles ont eu "du mal à faire face à l'imprévu », qui est le propre de la classe. Elles ont eu l'impression d'une grande prise de risque pour cette séquence. Elles ont peur «de dire des bêtises". Elles ont du mal à «s'accrocher à du solide », pour, par exemple, exploiter les erreurs des élèves, répondre aux questions nombreuses, et ainsi pratiquer un étayage efficace.

Tout ceci montre la difficulté que représente le rapport au savoir et renvoie à la question : que faut-il savoir et savoir-faire pour enseigner? Que faire de l'inconnu? Du risque de ne pas savoir?

\subsection{Comment les élèves s'emparent de la démarche}

Le retour des élèves a été très positif, quel que soit le collège et l'origine linguistique des élèves. La majorité d'entre eux a apprécié les deux séances, et les commente avec enthousiasme. Il est difficile de dire si cela vient de la nouveauté, qui les change des séances "habituelles", ou si pour eux il s'agit d'activités de fin d'années, qu'ils interprètent comme un projet, dans la mesure où les deux séances forment un tout. Une autre explication possible relève de l'importance accordée à leur travail par l'observation des chercheurs. Ils se sentent mieux écoutés et ont même des demandes d'explications à l'égard des chercheurs. 
29 Au grand étonnement des enseignantes, ils ont facilement trouvé l'ensemble des mots à chercher, à l'exception des élèves de $6^{\mathrm{e}}$ qui n'ont pas identifié le mot luni en roumain, équivalent du mot français mois (au pluriel). La phase de reconstruction ne leur a généralement pas posé problème. Des différences existent cependant entre les élèves de 6 ${ }^{\mathrm{e}}$ et les élèves de $4^{\mathrm{e}}$, le même scénario étant perçu comme plus difficile par les premiers, ce qui n'a rien de surprenant. Les élèves de $6^{e}$, même si certains d'entre eux ont perdu pied pendant la phase de reconstruction, ont cependant pu réaliser toutes les tâches, même s'ils ont été plus lents, et qu'ils ont eu davantage de mal à mettre en place une réflexion distanciée sur les mots.

Les élèves disent avoir découvert ce qu'étaient les langues romanes et leurs familles. Beaucoup d'entre eux ignoraient que les langues avaient des familles. Ils s'intéressent à l'histoire des mots qu'ils utilisent tous les jours. Ils se positionnent comme des chercheurs en herbe. On peut lire dans leurs réponses au questionnaire: «Je ne connaissais pas le protoroman, c'est chouette !», ou « Du coup, c'est plus simple d'apprendre une nouvelle langue en sachant ça!». Tous ont été mis en posture de recherche dans l'expérimentation. Ils ont été acteurs. La reconstruction relevait de leur action, concertée, conjointe ou individuelle selon les moments. Une enseignante note: "Ce qui est intéressant, c'est comment ils arrivent à rebondir d'une réflexion à l'autre et à créer collectivement quelque chose». Ils ont pu construire un savoir avec leurs pairs en contexte scolaire, ou élaborer un aspect des valeurs qui lui sont sous-jacentes.

31 Les élèves en difficulté scolaire, qui d'habitude prennent peu la parole, s'engagent davantage dans ce type d'activités, et ce d'autant plus qu'ils sont plurilingues. Il y a pour eux inconsciemment une mise en valeur de leurs savoirs et savoir-faire plurilingues. Ils sont en outre habitués à établir des ponts et à comparer, compétence rarement sollicitée dans l'enseignement « traditionnel » de la langue française.

\subsection{Stratégies et appuis pour une dynamique d'apprentissage}

32 À l'exception des élèves de la classe de $4^{\mathrm{e}}$ " défavorisée ", les élèves dans leur grande majorité savent généralement mobiliser leurs savoirs extrascolaires ou provenant d'autres disciplines pour résoudre les problèmes posés : le piccolo est un instrument de musique (italien), ils signalent une chanson apprise en espagnol, évoquent un jeu vidéo, parlent de la coupe du monde de football pour le portugais, reconnaissent le roumain par les "accents» sur les mots, etc. "Les enfants, constate une enseignante, ont une expérience des langues beaucoup plus riche qu'il y a 20 ou 30 ans ».

\subsection{Des savoirs provisoires}

Voici, à titre d'exemples, quelques-unes des réflexions des élèves, issues de leurs réponses au questionnaire rempli après l'expérimentation :

- «toutes les langues se ressemblent plus ou moins, mais en fouillant, on trouve des familles";

- les «mots français étaient plus compliqués avant, mais on découvre qu'ils peuvent venir d'autres langues »;

- certaines langues paraissent pour un élève «faciliter» les mots et donc « changer ou retirer des lettres » (l'italien), alors que d'autres comme l'espagnol et d'autres n'ont « pratiquement rien changé à la base du protoroman ». Ce qu'il veut dire, c'est que, contrairement à l'italien 
(qui aurait davantage "facilité ", c'est à dire supposons-nous simplifié les mots dans leur évolution), les mots de l'espagnol et d'autres langues n'auraient presque pas changé dans leur histoire ;

- «les langues de l'antiquité étaient parlées ou écrites», comme s'«il y avait plusieurs langues ». «C'est comme aujourd'hui »;

- " grâce à l'étymologie, on peut connaître beaucoup de choses et savoir l'origine des mots. Je ne pensais pas que les mots que j'utilise couramment avaient une histoire si longue »;

- "j'ai appris que les mots peuvent être raccourcis de leur racine ». Cet élève a saisi là une des lois de l'évolution des mots, celle d'économie.

34 Ces prises de conscience et découvertes des élèves se manifestent par des affirmations plus ou moins justes ou incomplètes par rapport au savoir enseigné. Ce sont des connaissances provisoires, en partie conscientisées et identifiées. Certaines semblent même déjà stabilisées. Un repérage des ces connaissances provisoires par l'enseignant serait utile pour appuyer ses conduites d'étayage vers des savoirs plus exacts ou plus complets.

En fin de parcours, un bilan écrit est élaboré sous la direction de l'enseignante. Cette phase de structuration des savoirs est essentielle, car elle permet de tisser des liens dans les découvertes successives des élèves, et de leur donner un statut d'importance pour l'enseignante dans le déroulé du cours. Lorsqu'un tel bilan manque, les élèves partent avec des savoirs fragiles, émergents, non conscientisés. Ici, les savoirs ont été discutés et, à tout le moins, mis en évidence, rendus visibles. Ils ont pris corps, se sont incarnés et ont été mis en relation avec le vécu des élèves. Puis ils ont été validés par l'enseignante, garante du contrat didactique.

\section{Conclusion}

Pour les observateurs, comme pour les enseignantes, les objectifs sont atteints : les élèves ont conscience que le français appartient à une famille de langues issues du latin; ils ont découvert des liens de parenté (distances et proximités) entre les langues et font preuve d'une plus grande ouverture à l'égard des langues romanes et des pays où elles sont parlées (notamment à l'égard du roumain, langue fortement dévalorisée par eux) ; même s'ils ont retenu que la seule règle de la mutation historique des mots était leur simplification (ce sur quoi insistait surtout le scénario, omettant les autres lois d'évolution), ils font le constat que les langues évoluent dans le temps ; enfin, il apparaît que la démarche de reconstruction est accessible à tous les niveaux de collège, elle n'est pas spécialement difficile, y compris en $6^{\mathrm{e}}$.

Les enseignantes se disent satisfaites de l'expérimentation et prêtes à poursuivre en ce sens. Elles sont surprises de l'enthousiasme des élèves et de leur engagement dans la tâche, qui se marque de manière tant verbale que non verbale. Les élèves ont apprécié une approche résolument collaborative.

Nous resterons prudents quant à ce succès, sachant à quel point une expérimentation met en valeur les expérimentateurs et induit un effet réussite. Par ailleurs, une telle entreprise ne pouvait aboutir sans l'aide du scénario fourni, sans l'appui des chercheurs, et sans formation spécifique des enseignants. Il demeure aussi quelques questions, notamment en termes de progression. En effet, les activités prévues devraient être mieux adaptées d'une part aux possibilités des élèves et d'autre part à leurs centres d'intérêt. 
Les élèves de $6^{\mathrm{e}}$ auraient eu besoin d'activités plus variées, notamment d'enregistrements sonores, pour mieux percevoir que les langues sont vivantes.

Il n'en reste pas moins que nous avons pu montrer par cette recherche interventionniste que l'approche comparatiste et reconstructionniste proposée par le DÉRom est tout à fait à la portée des élèves de collège, dès la $6^{\mathrm{e}}$, que des activités adaptées aux élèves sont nécessaires avec une vision curriculaire de l'étymologie, tout comme une vigilance à l'expression des représentations, des savoirs provisoires et à leur identification.

Tableau 9. Résultat de l'analyse sur le mot grand

\begin{tabular}{|l|l|l|l|l|l|l|}
\hline Français & Roumain & Italien & Catalan & Espagnol & Portugais & Protoroman \\
\hline grand & mari & grande & gran & grande & grande & grande \\
\hline
\end{tabular}

\section{BIBLIOGRAPHIE}

BUCHI, É. (2010). « Where Caesar's Latin does not belong: a comparative grammar based approach to Romance etymology ». In: Brewer, C. (éd.), Selected Proceedings of the Fifth International Conference on Historical Lexicography and Lexicology held at St Anne's College, Oxford, 16-18 June 2010. Oxford : Oxford University Research Archive. En ligne : http://ora.ox.ac.uk/objects/uuid\% 3A237856e6-a327-448b-898c-cb1860766e59.

BUCHI, É. \& SCHWEICKARD, W. (2011a). « Sept malentendus dans la perception du DÉRom par Alberto Vàrvaro ". Revue de linguistique romane 75, p. 305-312.

- (2011b). «Ce qui oppose vraiment deux conceptions de l'étymologie romane. Réponse à Alberto Vàrvaro et contribution à un débat méthodologique en cours ». Revue de linguistique romane 75 , p. 628-635.

CANDELIER, M. (dir.) (2003). L'éveil aux langues à l'école primaire. Evlang : bilan d'une expérimentation européenne. Paris : De Boeck.

CheVAllard, Y. (1985). La transposition didactique. Grenoble : Éd. La Pensée sauvage.

COSte, D., CASTELlOTTI, V. \& DUVERGER, J. (2008). Propositions pour une éducation au plurilinguisme en contexte scolaire. Paris : ADEB. En ligne :http://www.adeb.asso.fr.

DÉROM = BUCHI, É. \& SCHWEICKARD, W. (dir.) (2008). Dictionnaire Étymologique Roman (DÉRom). Nancy: ATILF. En ligne : http://www.atilf.fr/DERom.

ESCUDÉ, P. \& JANIN, P. (2008). Le point sur l'intercompréhension, clé du plurilinguisme. Paris: Clé international.

HAWKINS, E. (1984). Awareness of Language: An Introduction. Cambridge : Cambridge University Press. 
MACAire, D., Buchi, É., CARTON, F., CHAUVEAU, J. P., GReUb, Y \& HERBERT, C. (2014). « Un nouveau paradigme en étymologie romane à l'épreuve de la classe. Quelle pertinence pour des élèves de 11 à 14 ans ? » Enjeux 87, p. 49-72.

MACAIRE, D. (2008). « D'une didactique des langues à une didactique des plurilinguismes ? Réflexions pour la recherche ». Cahiers de l'Acedle 5, p. 1-46. En ligne : http://acedle.org.

MEISSNER, F.-J., MEISSNER, C., KLEIN, H. \& STEGMANN, T. D. (2004). EuroComRom - Les sept tamis. Lire les langues romanes dès le départ. Aachen : Shaker Verlag.

MOORE, D. (dir.) (1995). L'éveil au langage. Notions En Questions, Rencontre en didactique des langues. Paris : Éd. Didier.

VÀrVARO, A. (2011a). « Il DÉRom : un nuovo REW ? ». Revue de linguistique romane 75, p. 297-304. - (2011b). « La "rupture épistémologique” del DÉRom. Ancora sul metodo dell'etimologia romanza ». Revue de linguistique romane 75, p. 623-627.

\section{ANNEXES}

\section{Annexe : Fiche pratique destinée aux enseignants}

\section{Français grandes et ses cognats}

Les élèves partent de ce tableau construit par eux à partir de leurs découvertes dans les textes.

Tableau 1. Le mot grandes dans le texte en six langues

\begin{tabular}{|l|l|l|l|l|l|l|}
\hline Français & Roumain & Italien & Catalan & Espagnol & Portugais & Protoroman \\
\hline grandes & mari & grandi & gran & grandes & grandes & \\
\hline
\end{tabular}

Tableau 2. Guidage de l'activité et réponses

\begin{tabular}{|l|l|}
\hline Questions & Réponses \\
\hline $\begin{array}{l}\text { 1. Attention : vous vous êtes sûrement aperçus que dans ce tableau, il y a un } \\
\text { «intrus » : de quel mot s'agit-il? }\end{array}$ & $\begin{array}{l}\text { Il s'agit de } \\
\text { roumain mari. }\end{array}$ \\
\hline $\begin{array}{l}\text { 2. En effet, roumain mari ne remonte pas au même étymon que français } \\
\text { grandes et les autres mots que vous avez reportés dans le tableau. Cela ne doit } \\
\text { pas trop nous étonner: s'il est vrai que le roumain est bien une langue } \\
\text { romane à part entière, dont l'origine se trouve, comme pour le français, dans } \\
\text { la variété parlée du latin, cette langue vit depuis longtemps déjà sa vie } \\
\text { géographiquement séparée des autres langues romanes, de sorte qu'elle a } \\
\text { évolué de façon originale. Qu'est-ce que nous pourrions faire pour ne pas } \\
\text { oublier que mari remonte à un autre étymon que grandes? }\end{array}$ & $\begin{array}{l}\text { lableau. } \\
\text { tans le }\end{array}$ \\
\hline
\end{tabular}


Tableau 3. Le mot grandes après le travail d'analyse en six langues

\begin{tabular}{|l|l|l|l|l|l|l|}
\hline Français & Roumain & Italien & Catalan & Espagnol & Portugais & Protoroman \\
\hline grandes & mari & grandi & gran & grandes & grandes & \\
\hline
\end{tabular}

Tableau 4. Conceptualisation de la marque du pluriel en six langues

\begin{tabular}{|l|l|}
\hline Questions & Réponses \\
\hline $\begin{array}{l}\text { 3. Français grandes est un (féminin) pluriel. En travaillant sur ans et ses } \\
\text { correspondants, nous avons vu que les langues romanes connaissent } \\
\text { essentiellement deux manières de former le pluriel (des noms et des adjectifs). } \\
\text { Vous souvenez-vous de ces deux procédés? }\end{array}$ & $\begin{array}{l}\text { - Ajout de -i }-s . \\
\text { [au radical]. }\end{array}$ \\
\hline $\begin{array}{l}\text { 4. En nous appuyant sur cette information, nous allons à présent étiqueter } \\
\text { chaque mot du tableau en « singulier » ou "pluriel ». Dictez-moi! }\end{array}$ & $\begin{array}{l}{[C f . \quad \text { tableau }} \\
\text { ci-dessous. }]\end{array}$ \\
\hline
\end{tabular}

Tableau 5. Reconnaissance de la marque du pluriel à partir des textes en six langues

\begin{tabular}{|l|l|l|l|l|l|l|}
\hline Français & Roumain & Italien & Catalan & Espagnol & Portugais & Protoroman \\
\hline grandeS & & grandI & gran & grandes & grandeS & \\
PL & & PL & $S G$ & PL & PL & \\
\hline
\end{tabular}

Tableau 6. Analyse du catalan et réponses

\begin{tabular}{|c|c|}
\hline Questions & Réponses \\
\hline $\begin{array}{l}\text { 5. Nous sommes étonnés par le singulier du catalan. Mais en se reportant au } \\
\text { texte, on s'aperçoit que ce singulier apparaît dans le syntagme la gent gran (/la } \\
3 \varepsilon \text { En 'gran/), littéralement 'la gent grande', qui traduit les grandes personnes. En } \\
\text { français, gens, le « cousin » de catalan gent, est surtout utilisé au pluriel, mais } \\
\text { dans certains emplois littéraires et un peu archaïques, gent peut apparaître au } \\
\text { singulier en français aussi. Connaissez-vous des emplois de ce type? }\end{array}$ & $\begin{array}{l}\text { - La gent } \\
\text { masculine 'les } \\
\text { hommes'; } \\
\text { - la gent } \\
\text { canine 'les } \\
\text { chiens'; } \\
\text { - la gent qui } \\
\text { porte crête 'les } \\
\text { coqs' (La } \\
\text { Fontaine) etc. }\end{array}$ \\
\hline
\end{tabular}


6. Il est bien clair que l'adjectif singulier du catalan et les adjectifs pluriels des autres langues romanes ne peuvent pas remonter exactement au même étymon (mot-origine). D'autre part, ces adjectifs sont au féminin, car ils déterminent personnes et ses traductions. Pour simplifier les choses, et aussi pour travailler sur les variantes les plus neutres de ces adjectifs - leur forme de référence, celle qui apparait comme entrée dans les dictionnaires -, nous allons remplacer les adjectifs du tableau par leur correspondant masculin singulier. Pouvez-vous m'aider à faire cela?

Tableau 7. Le mot grand après le travail d'analyse du texte en six langues

\begin{tabular}{|l|l|l|l|l|l|l|}
\hline Français & Roumain & Italien & Catalan & Espagnol & Portugais & Protoroman \\
\hline grand & grande & gran & grande & grande & \\
\hline
\end{tabular}

Tableau 8. Étude des formes et scénarios possibles sur l'ancêtre du mot grand

\begin{tabular}{|c|c|}
\hline Questions & Réponses \\
\hline $\begin{array}{l}\text { 7. On constate que trois formes s'opposent: (1) italien, espagnol et } \\
\text { portugais grande, (2) français grand et (3) catalan gran. } \\
\text { Hypothétiquement, on peut donc imaginer deux scénarios : soit } \\
\text { italien, espagnol et portugais grande représentent la forme } \\
\text { originelle, que le français et le catalan ont abrégée au cours du } \\
\text { temps, soit catalan gran représente la forme originelle, que les } \\
\text { autres langues romanes ont petit à petit allongée avec le temps. } \\
\text { Pouvez-vous, en utilisant le même raisonnement que celui que nous } \\
\text { avons employé pour reconstruire protoroman anni, puis } \\
\text { protoroman dormire, départager ces deux hypothèses? }\end{array}$ & $\begin{array}{l}\text { - Oui. } \rightarrow \text { Tout va bien. } \\
\text { - Non. } \rightarrow \text { Refaire le même } \\
\text { raisonnement que pour } \\
\text { ans et ses cognats, } \\
\text { questions } 4 \text { à } 7 \text {. }\end{array}$ \\
\hline
\end{tabular}

$\rightarrow$ Cela nous permet de reconstruire l'ancêtre commun de français grand et de ses « cousins » :

\section{NOTES}

1. Projet de réforme de l'enseignement au collège, 2015 : les EPI, destinés aux élèves à partir de la classe de cinquième, sont conçus comme des travaux interdisciplinaires en petits groupes (trois heures d'EPI par semaine). Huit thèmes sont au choix, six d'entre eux au minimum sont à étudier entre la cinquième et la troisième : « développement durable ", « information, communication et citoyenneté ", "langues et cultures de l'antiquité », "langues et cultures étrangères ou régionales ", «monde économique et professionnel ", " corps, santé, sécurité », "culture et création artistiques » et "sciences et société ».

2. Parmi elles, on peut citer Galatea, EuroCom4, ou encore les Parcours romans.

3. L'un des projets d'envergure a été le projet européen Evlang, piloté par M. Candelier.

4. 3 classes de $6^{\mathrm{e}}$ (en juin de l'année scolaire) et 4 classes de $4^{\mathrm{e}}$. 
5. À titre d'exemple, voici la première phrase de cette page, en six langues romanes :

- Lorsque j'avais six ans j'ai vu, une fois, une magnifique image, dans un livre sur la Forêt Vierge qui s'appelait « Histoires Vécues ».

- Odată, pe vremea când aveam eu şase ani, am dat peste o poză minunată, într-o carte despre pădurile virgine, numită « Întâmplări trăite ».

- Quan tenia sis anys, vaig veure una vegada un magnífic dibuix en un llibre sobre la Selva Verge que es deia « Histories viscudes ».

- Cuando tenía seis años, vi una vez una lámina magnífica en un libro sobre el Bosque Virgen que se llamaba « Historias Vividas ».

- Un tempo lontano, quando avevo sei anni, in un libro sulle foreste primordiali, intitolato "Storie vissute della natura ", vidi un magnifico disegno.

- Certa vez, quando tinha seis anos, vi num livro sôbre a Floresta Virgem, "Histórias Vividas ", uma imponente gravura.

6. Propos extraits de la séance e/ de « debriefing » indiquée en 3.3.

\section{RÉSUMÉS}

Une recherche-action dans plusieurs classes de collège en Lorraine montre que l'approche comparatiste entre langues romanes et reconstructionniste (recherche des étymons en protoroman) proposée par les spécialistes de linguistique historique de l'équipe DÉrom (ATILF) est à la portée d'élèves de collège. Elle permet à ceux-ci, mis en position de chercheurs, de prendre conscience que le français appartient à une famille de langues issues du latin, des liens de parenté entre ces langues, et de certaines règles de l'évolution des langues dans le temps. La démarche de reconstruction de certains étymons leur est accessible, elle suscite leur intérêt et leur permet de s'ouvrir aux langues et à leur histoire. Le paradigme en étymologie apporté par le DÉrom donne lieu à une vision plus juste de l'histoire du français et peut contribuer à un déplacement des représentations sur l'étymologie dans le discours institué.

An action-research project conducted in several secondary schools (age 11-15) in Lorraine by specialists in historical linguistics from the Atilf's DÉRom group shows that Romance languages can successfully be introduced at this level via a comparative approach including reconstruction of etymons in Proto-Romance. Treating learners as researchers sensitises them to the position of French in a family of language stemming from Latin, to the connections between these languages, and to a number of rules governing the evolution of languages over time. The reconstructive work on certain etymons is within their capabilities, motivates them and opens them up to languages and their history. The etymological approach provides a more accurate picture of the history of the French language and can successfully contribute to a shift in representations towards etymology.

\section{INDEX}

Keywords : etymology, secondary school, didactic transposition, Romance languages, Vulgar Latin

Mots-clés : étymologie, collège, transposition didactique, langues romanes, latin vulgaire 
AUTEURS

FRANCIS CARTON

DOMINIQUE MACAIRE 